be followed by a slight gasp or respiratory effort, which encouraged us to still further efforts; and we worked on until we were exhausted, and at last he breathed and the pulse came, and he returned from death to life. The father and mother were waiting in the next room to hear the result of the operation, little thinking of the strife in which we were engaged. If they had been present the scene would have been terrible, and I believe the patient would have died. This man was healthy in every respect ; no sickness, no disease ; in fact, he called on me in Clifton a fortnight afterwards, looking perfectly well, and requiring advice about an artificial eye. He did not know, until I told him, that he had run such risks; and yet he had most decidedly been killed by a small dose of chloroform, with this little exception, that he did not die, but our exertions saved him. Without a third pair of hands he must have died : I never saw a person so corpse-like come to life again.

These and numberless other instances prove that there are without doubt two sets of cases in which lives are endangered or lost-viz., where the heart's action is stopped, and when the breathing ceases. To see the struggles and congested state of many patients while chloroform is being administered, the wonder is that we cannot add to these two a third series, where death occurs from apoplexy or some cerebral damage ; and I have seen many instances of lengthened operations on persons severely hurt, where they have not died at once, but have lingered in a half conscious state for a day or two and then died. do not remember this class of case as having occurred, at any rate so frequently, before the days of chloroform, and they are not unfrequently in reality deaths from chlroform, affecting the brain in patients so depressed by disease or accident that they cannot get rid of the drug. In private practice we have no sufficient means of testing a question of this kind, and I should like to draw the attention of some of our acting hospital surgeons here present to its solution.

I have in the cases above narrated sufficiently indicated the treatment to be pursued in a similar emergency : a battery, the wet towel, the Silvester method, and the drawing forward of the tongue to relieve the larynx, seem to be the only reliable means.

I must agree, of course, with the universally received opinion that the artificial induction of anzesthesia is a great boon to mankind; but I am not opinion that it is a very safe one, nor that chloroform is its safest medium. I have for many years used a mixture of chloroform and ether, two of the latter to one of the former, under the impres. sion that it is safer than pure chloroforn. I think, too, that care in the administration of anresthetics, and due preparations for restoring life in cases of danger, are important elements in the successful use of these agents ; but I also believe that the anæsthetic state itself is dangerous, and that if you were to take 2,500 sick or wounded, or weak and diseased persons, and make them all so drunk with wine or brandy that they could neither feel nor move, a certain considerable percentage would never wake up to life again in this world. We are repeatedly urged to admit to our patients that chloroform is quite safe, but this I never will nor can do.

I have often tried, but I fear hitherto in vain, to impress on my in firmary colleagues and other professional friends, that the meaning of the word anresthesia is the state where no pain is felt, and that they have no right in every case to push it so far as to destroy the power of motion for their own comfort in operating. If the patient cannot feel, that is all that he wants ; your assistants must keep him quiet by force of arms.

The lithotomy position, with the hands and feet strapped together, is very unfavourable for chloroform, because of the fixed state of the chest; and I have seen some very uncomfortable cases from this cause. Chloroform and other anzesthetics act on young children fairly; but the little patients are apt to fall into a deep stupor after the operation has been finished, if it be not very long, and I have had not unfrequently considerable, but probably uncalled for, anxiety from this cause. Before the time of chloroform, patients made up their minds to necessary operations as readily, I believe, as they do now. It is a common and popular, but most erroneous idea, first started by Professor Miller, that the use of anxsthetics affords great relief to the operator as well as to the patient. Never was a greater mistake. It is a mere sentiment, likely to catch the mind of the public, and to be brought forward as a sign of the great tenderness of the surgeon's heart; but I must say that my own opinion agrees with that of $\mathrm{Mr}$. Erichsen, which I saw quoted some time ago-viz., that the use of chloroform, at any rate in private practice, adds at least a hundred per cent. to the anxieties and responsibilities of the surgeon. In serious or delicate operations, such as ligature of arteries, or removal of the eyeball, or lithotomy, or hernia, it is a great evil to divide the operator's attention between the state of the patient from the chloroform and his own manipulations. In one of my earliest operations for removal of the eyeball, I was told afterwards that at one period of the operation the patient had uttered a piercing shriek; but I was so much in an anæsthetic state, as far as hearing was concerned, from my attention to my own share in the proceedings, that I did not hear it.

Although it is presumptuous in such an assembly as this for me to touch on the midwifery question, I will nevertheless give my experience, which has been little, but very satisfactory. I have mixed about two drachms of chloroform with six of eau de Cologne, and given it, with a minim-glass armed with a cork, to an assistant, with the direction to keep ten minims in the glass, and to pour this quantity as often as I direct on the handkerchief held by the patient. This she presses over her mouth and nose; and if it be repeated at every pain as soon as it becomes necessary, and more frequently at the last pains, the sufferings of childbirth are relieved without risk. She takes only about two and a half minims of chloroform at each dose, and with this I have known a patient delivered in a state of complete unconsciousness.

In conclusion, while we are ready to give our patients the benefit of anæsthetics, and to take every possible precaution and care against any mishap, I think the entire responsibility shhould rest on the public who call for their use, and who choose to run a risk of life in cases where the operation itself and its consequences are entirely free from danger : and, practically, the public does take the blame; at any rate, the stereotyped verdict, in fatal cases, is "Death from chloroform, but no blame is attached to the medical men."

\section{THE ORIFICES OF THE UTERUS AND THEIR SURGICAL TREATMENT.}

BY J. HENRY BEN NET, M.D., Late Obstetric Physician to the Royal Free Hospital, London.

Is Dr. Matthews Duncan's last communication on the above subject, he dismisses my criticism on his former papers in a very few words, merely stating that $I$ have misunderstood and misrepresented him, and that $\mathrm{I}$ am wrong anatomically, physiologically, and pathologically in my views respecting the uterine orifices. I may mention, what my old and esteemed friend Dr. Duncan does not, that the words which he used constitute the title of the paper I read last August at the meeting of the British Medical Association at Birmingham, which was published in the British Medical Journal the following month. I would add that, great as is the authority of Dr. Duncan, it is not sufficiently great to stamp, by a mere assertion, as a tissue of errors an elaborate paper, deliberately written, founded on thirty-five years' experience, and brought before the profession with a view to debate and discussion, and to prevent uterine surgery from running riot, as I conscientiously believe it now is doing.

My criticism of Dr. Matthews Duncan's scientific and interesting papers is this. Throughout his exhaustive discussion of the mechanical power, of the actual force used in the various modes of dilating the cervical canal, and especially the os internum, he never once even alludes to the nature of the obstruction which he is endeavouring to dilate or overcome. What is meant by all this surgical cutting and dilatation? What is the pathological condition which the dilatation or force is meant to overcome? What is the pathological condition that resists so as to bend a strong silver sound, or which can only be overcome by a pressure of four pounds? We know what a stricture is in the male urethra, the fibrous gristly nature of the contraction, and the antecedent pathological conditions; we know that, if we resort to forcible sounding or catheterism, we break through these fibrous gristly structures, and that without probably using a pressure of four pounds. What I wish to know is, what is the actual pathological condition of the cervical canal or of its orifices, external or internal, which leads one physician of eminence in London to divide the os internum with a double metrotome three hundred times in two years in his consultingroom, and another in New York to divide the os externum and entire cervix down to its vaginal attachment in five hundred cases in the same period of time? Indeed, I come upon traces of these operations constantly in my practice in all kinds of cases, without finding a clue to the motive that dictated them. These serious operations, not to speak of forcible dilatation of all kinds, appear to me, judging from what $I$ see and hear, to be rashly, blindly carried out, as a kind of surgical panacea for anything and everything-sterility, dysmenorrhœa, displacements, etc.

This wild confusion in practice-this constant recourse to severe surgical treatment and operations in women-originates, in my opinion, in the failure to recognise the facts which I laid down in the second edition of my work on Uterine Inflammation in 1848 , twenty-five years ago. I therein say and now repeat : I. That, if the os externum will admit a 
good sized sound, say, No. 6 or 8 , it is an absurdity to divide it on any grounds ; 2 . That the cervical canal between the os externum and the os internum is an infundibuliform cavity, and seldom or never is contracted or requires dilatation, except in congenital cases, or in cases where there are adhesions from the action of caustics or of labour; 3 . That the os internum, so far from being open, as described in anatomical works, is closed by a vital contraction of the circular fibres, like the anus, and so closed that, except in morbid conditions, force has to be used with the sound in perfectly healthy women; the exception being certain morbid conditions, and in some physiological ones, spe cified in my paper read at the British Medical Association.

I think the non-recognition of this anatomical and physiological fact is at the bottom of all the rash useless surgery which I deprecate, and that I am only doing my duty in bringing the subject before the profession from my semi-retirement on the shores of the Mediterranean. Surely, as one of the Nestors, I may say, of modern gynæcology, as one who long fought the battle of uterine progress single-handed, I have a right to be heard on the subject. Nor do I think that my junior friends in the gynæcological field should feel hurt if in doing so I go counter to their views and opinions. The world of science is open to all. I have fought many a hard battle with a smiling face and a hand held out to my antagonists. Moreover, we are all responsible for our opinions uttered publicly in societies, or published in the medical journals, and must stand or fall by them.

I do not enter into any further details on this important subject, for my paper is there-in the number of this JoURNAL for September $\mathbf{2} \mathbf{I}-$ to be referred to. I would only ask every gynæcologist who reads this statement to examine carefully with the sound every case of uterine or vaginal disease, slight or severe, that he sees; and, with the exceptions named in my memoir, he will surely find that the sound stops at one inch and a half from the external os, the entire depth of the uterine cavities being two inches and a half. This is the depth at which the sound should pass-two and a half inches-if it enter the uterine cavity, passing the os internum.

When we consider that every healthy woman examined with the sound -if I am right, and if it stop physiologically at the os internummust be considered to suffer from a morbid contraction by those who are unacquainted with the fact, it becomes evident at once to what a frightful excuse for useless surgery such ignorance leads. Every healthy woman in the kingdom examined under this impression may be pronounced to have contraction, and be dilated or incised, according to the mode of practice adopted.

When we consider, too, that, according to the census, half a million of married women are sterile (one in six), we see to what such ignorance must lead. The existence of the natural sphincter in this half million of women justifies, in those who ignore it, any wild fancy, any amount of dilatation or of division.

I do not think there is just now a more important question before the profession; and I sincerely trust that Dr. Matthews Duncan's memoir will lead to its thorough discussion and elucidation, by himself first, and then by others. I may add that such a discussion should follow the order I have given, and be anatomical, physiological, and pathological..

Mentone, France, February 9th, 1873 .

\section{NOTE ON THE CHEMICAL HISTORY OF THE ERUPTION SOMETIMES FOLLOWING THE ADMINISTRATION OF CHLORAL.*}

\section{By T. P. BLUNT, M.A., F.C.S}

I Do not think that I shall justly incur the stigma of going beyond my last, if $I$ offer a suggestion with regard to the origin of this phenomenon, now tolerably familiar to practitioners, a strongly marked case of which came under my immediate observation a few years ago, before it was well known and recognised. Although, therefore, an explanation at once suggested itself, I hesitated to call attention to it, thinking it just possible that the exception might be the result of a unique idiosyncracy.

I must first remind you of the reaction to which chloral hydrate owes its physiological effect. When chloral or "chloraldehyde" contact with an alkali, chloroform is produced, together with formiate of the alkali. The first named substance is very easily detected in a mixture of the kind, from which it separates with all its characteristic properties; the latter is not so readily recognised, though its presence is, of course, equally invariable. When a dose of chloral is taken into

* Read before the Shropshire Scientific Branch. the stomach and absorbed into the circulation, it meets with free alkali in the blood, the reaction described follows, and the chloroform produced exercises its usual hypnotic action upon the patient. Under normal conditions, the formiate produced is no doubt eliminated by the kidneys, after the manner of most other soluble salts ; but it must be borne in mind that it is in the meanwhile carried about in the circulation, diffusing itself through the whole body, and therefore finding its way into the capillaries of the skin, and into the neighbourhood of the sweat-glands.

Now the sweat is always during health more or less acid, and physiological chemists tell us that the acidity of the secretion is usually derived from lactic acid-a powerful acid, somewhat analogous to the tartaric; it is probable, therefore, that when the alkaline formiate comes into contact with it, formic acid would be set free. Under normal conditions, the amount of acid might be too small to produce any unpleasant effects ; but when the sweat is abnormally acid, as is often the case throughout life with certain individuals, and under exceptional circumstances might occur to any one, the amount liberated might be sufficient to exercise the characteristic irritant action familiar to us in the sting of the ant and the nettle. Two probable objections to this theory present themselves-the first founded on the dilution of the acid in the liquor sanguinis, the second upon its minute quantity. My reply to the first objection is, that the boiling point of formic acid being about 220 deg. Fahr. -i.e., higher than that of water, it would probably be concentrated in the sweat-glands by insensible perspiration. To the second I answer by pointing to the considerable amount of irritation following the injection of the contents of a single poison gland of the common nettle, which is an object so minute as to be scarcely discernible without the microscope. I think, therefore, that I have made out a tolerable case against the alkaline formiate, which is the complementary product of the reaction by which chloroform is produced upon the administration of chloral hydrate.

\section{REPORTS}

or

\section{MEDICAL AND SURGICAL PRACTICE IN THE HOSPITALS OF GREAT BRITAIN.}

\section{ST. BARTHOLOMEW'S HOSPITAL.}

A CASE OF SECONDARY HAMORRHAGE FROM THE EXTERNAL CAROTID ARTERY : WITH REMARKS.

(Under the care of Mr. SAVORY.)

WM. EALES, aged 48, was admitted on the 23 rd of last June for a tumour in the right side of his neck. He first noticed it about two months previously, when it was of the size of a small filbert; but up to the time of his admission it had steadily enlarged, and was then of the size of a small orange. It was uniformly elastic, and fluctuated on pressure. It was situated beneath the sterno-mastoid, and that muscle could be plainly traced curving over it. A distinct pulsation could be felt by the hand placed on it, and a bruit might be heard on applying a stethoscope to the surface, but this was obviously transmitted from the subjacent artery. His voice, for the last fortnight, had become very husky, and he suffered from considerable pain in the back of his head. There was no difficulty in swallowing, and his general health was tolerably good. A consultation was held, at which some difference of opinion was expressed as to the contents of the tumour, but it was unanimously agreed that previously to any operation a fine trocar should be passed. This was done on July $5^{\text {the }}$ and a few ounces of thin sanguinolent fluid were evacuated. On July 8th, the patient being under chloroform, the cyst was freely laid open. It was lined throughout by a smooth mem. brane, and extended irregularly among the deep structures of the neck. On introducing the finger, the carotid artery could be plainly felt through its wall pulsating at the bottom of the wound. Strips of lint were in troduced and changed daily for about a week, the wound being gently washed out by a syringe with a weak solution of Condy's fluid, as the discharge became offensive. Little or no constitutional disturbance followed the operation; the thermometer did not register above $98 \mathrm{deg}$. in the axilla.

About midday on July 17 th, hæmorrhage so sudden and profuse occurred that he lost, as it was calculated, over three pints of blood in little more than a minute. A larger stream of blood, having nearly the calibre of one's little finger, was seen to spout from the wound, but was immediately controlled by digital pressure. He was at once taken into the theatre, and under chloroform the original incision was extended, and the greater portion of the cavity exposed. The part around the 